\title{
Mathematical model of photoacoustic microscopy with piezoelectric detection
}

\author{
E.V. Vertsanova, Yu.I. Yakimenko \\ Kiyv National Technical University «KPI», 37, prospekt Peremogy, 252056 Kyiv, Ukraine
}

\begin{abstract}
Absract. Mathematical model is formulated for piezoelectric detection of the photoacoustics effect in optically semitransparent and thermally thick object with subsurface non-homogeneity in the case of free holding a piezodetector in a photoacoustic cell of the respective microscope. The model enables to estimate the influence of subsurface defects on the photoacoustical signal and to take into account the contribution of this factor into output signal of investigated objects.
\end{abstract}

Keywords: non-destructive check, photoacoustic microscopy, subsurface defect, piezotransducer.

Paper received 09.09.99; revised manuscript received 08.10.99; accepted for publication 18.10.99.

In recent years photoacoustic microscopy methods (PAM) with a piezoelectric signal detection are widely used as a non-destructive method of checking the material quality and products of electronic industry.

PAM is based on a phenomenon of generation and spreading of both thermal and acoustic waves excited by modulated in intensity laser radiation, $I$. The absorbed radiation heats the enlightened area (Fig.1), and the periodic thermal fluxes $Q_{t}$ spreading in the object appear.

Owing to periodic heating and thermal deformation, acoustic waves with the same frequency as the thermal ones appear and spread within a sample. The acoustic oscillations appeared in the object are detected by a piezosensor. The rise and spread processes of both thermal and acoustic waves depend on the local area properties near a point of excitation. Therefore, it is possible to get a spatial distribution of the undersurface area properties by moving a laser beam along the sample surface and synchronously detecting a photoacoustic signal [1-3].

To detect the photoacoustic signal (PAS) a piezoelectric method based on the direct detection of an acoustic wave in the object is often used as the most sensitive one [4-6].

Now, there is no any common and universal model of a photoacoustic effect (PAE), which makes it difficult to decode photoacoustic images. Usually some assumptions are accepted and simplified models are proposed for concrete experimental conditions. For example, in condensed media the Jackson-Amer model is widely used for interpretation PAE with a piezoelectric signal detection [2]. However, it is valid only for homogeneous samples while the ones with the inner inhomogeneities are usually interesting in the most of practical cases. The same deficiency is also habitual to the piezoelectric detection model elaborated by A. I. Morozov [5]. Therefore, required is a further development of the mathematical model which would allow one to describe more adequately results of testing nonhomogeneous objects using the PAM.

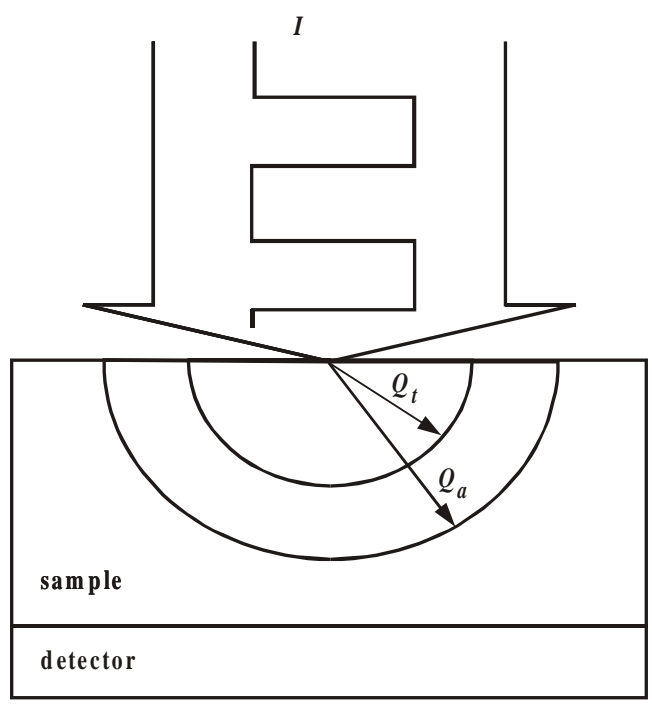

Fig. 1. Scheme of the photoacoustic effect in solid. 


\section{E.V. Vertsanova, Yu.I. Yakimenko: Mathematical model of photoacoustic microscopy...}

\section{Theoretical model}

An infinite many layer system (Fig.2) modeling the isotropic crystal with impurity centres was studied. To calculate PAS values detected by a piezoelectric transduccer of the longitudinal bulk waves, which has the acoustic contact with the object, full thermoelasticity equations in one-dimensional geometry were solved with account of the following assumptions:

1) sample is optically opaque (the absorption of radiation is described by the exponential law);

2) sample is optically and thermally thick;

3 ) the heat exchange with air on the object surface is neglected;

4) the contact of the sample surface with a piezoelectric transformer is considered as the ideal one and acoustic reflection from the interface is neglected;

$5)$ sample is thermally and acousticaly homogeneous on each interface;

6) mechanical strains and deformations are continuous;

7) interfaces at $x=0$ and $x=l_{1}$ are free.

PAE consists in that the investigated object is periodically heated under irradiation with the intensity modulated in accord to expression $I=\frac{I_{0}}{2}(1+\cos \omega t)$. Such heating causes acoustic oscillations in a sample. These oscillations are detected by piezoelectric longitudinal bulk waves transducer whose electrical signal gives information on both optical and thermoelastic object properties. The open-circuit voltage $V$ on the piezosensor plates is proportional to a difference of the bias amplitudes $u$ on its boundaries:

$V=h\left(u_{l_{1}}-u_{l}\right)$,

where $h=\frac{e}{\varepsilon^{S}}, e$ is a piezomodulus, $\varepsilon^{S}$ is a dielectric constant of the squeezed crystal.

Therefore, PAE calculation with the piezoelectrical detection is reduced to an evaluation of the difference of bias amplitude displacements on the piezosensor boundaries. For this purpose it is necessary to solve jointly equations of a thermal conduction and wave equations for the sample with the sizes $0 \leq x \leq l$, for the inhomogeneity with the sizes $h \leq x \leq h_{1}$, and for the piezosensor with the sizes $l \leq x \leq l_{1}$.

Let us enter the following designations:

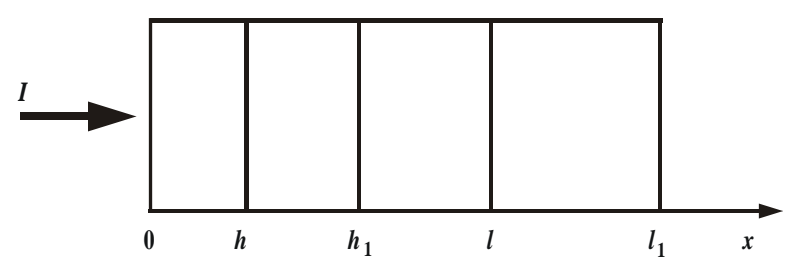

Fig. 2. Geometry of the task of PAE piezoelectrical detection. $0 \leq x \leq l$ are the sample sizes, $h \leq x \leq h_{1}$ are the defect sizes (model layer), $l \leq x \leq l_{1}$ are the piezotransducer sizes.

$$
\delta_{1}=h ; \quad \delta_{2}=h_{1}-h ; \quad \delta_{3}=l-h_{1} ; \quad \delta_{4}=l_{1}-l .
$$

The indexes $j=1 \ldots 4$ used also for others parameters in the text are related to these boundaries.

For $0 \leq x \leq h$ :

$\frac{1}{\chi_{1}} \frac{\partial \theta_{1}}{\partial t}=\frac{\partial^{2} \theta_{1}}{\partial x^{2}}+\frac{\beta I_{0} e^{i \omega t}}{2 \kappa_{1}} e^{-\beta_{1} x}$,

$\frac{\partial^{2} u_{1}}{\partial x^{2}}-\frac{1}{v_{1}^{2}} \frac{\partial^{2} u_{1}}{\partial t^{2}}=\eta_{1} \alpha_{1} \frac{\partial \theta_{1}}{\partial x}$

For $h \leq x \leq h_{1}$ :

$$
\begin{aligned}
& \frac{1}{\chi_{2}} \frac{\partial \theta_{2}}{\partial t}=\frac{\partial^{2} \theta_{2}}{\partial x^{2}}+\frac{\beta I_{0} e^{i \omega t}}{2 \kappa_{2}} \beta_{2} e^{-\beta_{1} x} e^{-\left(\beta_{1}-\beta_{2}\right) \delta_{1}} \\
& \frac{\partial^{2} u_{2}}{\partial x^{2}}-\frac{1}{v_{2}^{2}} \frac{\partial^{2} u_{2}}{\partial t^{2}}=\eta_{2} \alpha_{2} \frac{\partial \theta_{2}}{\partial x}
\end{aligned}
$$

For $h_{1} \leq x \leq l$ :

$$
\begin{aligned}
& \frac{1}{\chi_{1}} \frac{\partial \theta_{3}}{\partial t}=\frac{\partial^{2} \theta_{3}}{\partial x^{2}}+\frac{\beta I_{0} e^{i \omega t}}{2 \kappa_{1}} \beta_{1} e^{\left(\beta_{1}-\beta_{2}\right) \delta_{2}-\beta_{1} x}, \\
& \frac{\partial^{2} u_{3}}{\partial x^{2}}-\frac{1}{v_{1}^{2}} \frac{\partial^{2} u_{3}}{\partial t^{2}}=\eta_{1} \alpha_{1} \frac{\partial \theta_{3}}{\partial x} .
\end{aligned}
$$

For $l \leq x \leq l_{1}$ :

$\frac{\partial^{2} u_{4}}{\partial x^{2}}-\frac{1}{v_{4}^{2}} \frac{\partial^{2} u_{4}}{\partial t^{2}}=0$

where $\theta_{j}$ is a temperature; $u_{j}$ is a bias; $\alpha_{j}$ is a coefficient of the cubic expansion; $v_{j}$ is a spreading velocity of the longitudinal bulk waves; $\eta_{j}=\frac{B}{c_{j}{ }^{T}}, B$ is a bulk cofficient of elasticity; $c_{j}{ }^{T}=\lambda+2 \mu, \lambda$ and $\mu$ are the Lame coefficients.

Equations (1) - (7) are solved with the following boundary conditions:

On the interface «air - sample» $x=0$ :

$\frac{\partial \theta_{1}(0)}{\partial x}=0$

$T_{1}(0)=0$.

On the right side of the interface «homogeneous layer defect»:

$\theta_{1}(h)=\theta_{2}(h)$

$\kappa_{1} \frac{\partial \theta_{1}(h)}{\partial x}=\kappa_{2} \frac{\partial \theta_{2}(h)}{\partial x}$

$T_{1}(h)=T_{2}(h)$, 


\section{E.V. Vertsanova, Yu.I. Yakimenko: Mathematical model of photoacoustic microscopy...}

$u_{1}(h)=u_{2}(h)$.

On the back side of the interface «defect - homogeneous layer»:

$$
\begin{aligned}
& \theta_{2}\left(h_{1}\right)=\theta_{3}\left(h_{1}\right), \\
& \kappa_{2} \frac{\partial \theta_{2}\left(h_{1}\right)}{\partial x}=\kappa_{3} \frac{\partial \theta_{3}\left(h_{1}\right)}{\partial x} \\
& T_{2}\left(h_{1}\right)=T_{3}\left(h_{1}\right), \\
& u_{2}\left(h_{1}\right)=u_{2}\left(h_{1}\right) .
\end{aligned}
$$

On the interface «sample - piezo-sensor»:

$$
\begin{aligned}
& \theta_{3}(l)=0, \\
& T_{3}(l)=T_{4}(l), \\
& u_{3}(l)=u_{4}(l),
\end{aligned}
$$

On the piezosensor free surface:

$$
T_{4}\left(l_{1}\right)=0 \text {, }
$$

where $k_{j}$ is a thermal conductivity; $T_{j}$ are the strains.

Formulating these requirements it was considered that the boundary conditions are the mathematical formulation of the proposed assumptions, namely, that the heat exchange on the interface of air with the object can be neglected (8), the object is thermally thick (11), the mechanical strains and deformations are continuous (9b), (9c), (10b), (10c), (11a), (11b), and the boundaries $x=0$ and $x=l_{1}$ are free (8a), (12). lows:

The strains $T_{j}$ are related to the deformations $u$ as fol-

$$
\begin{aligned}
& T_{1}(x)=c_{1}^{T} \frac{\partial u_{1}}{\partial x}-B_{1} \alpha_{1} \theta_{1}, \\
& T_{2}(x)=c_{2}^{T} \frac{\partial u_{2}}{\partial x}-B_{2} \alpha_{2} \theta_{2}, \\
& T_{3}(x)=c_{1}^{T} \frac{\partial u_{3}}{\partial x}-B_{1} \alpha_{1} \theta_{3}, \\
& T_{4}(x)=c^{D} \frac{\partial u_{4}}{\partial x},
\end{aligned}
$$

where $c^{D}=c^{E}\left(1+\frac{e^{2}}{\varepsilon^{S} c^{E}}\right), c^{E}$ is the coefficient of rigidity of the piezoelectric.

At the harmonic excitation of sample surface by the intensity modulated light

$I=\frac{I_{0}}{2}(1+\cos \omega t)$

the temperature and strain distributions in the sample looks like:

$\theta(x, t)=e^{i \omega t} \hat{\theta}(x)$, $u(x, t)=e^{i \omega t} \hat{u}(x)$.

Omitting cumbersome evaluations we shall write down for the open-circuit voltage on the piezotransducer an expression obtained by the solution of the equation system (1) - (7):

$V=h\left[u_{4}\left(l_{1}\right)-u_{4}(l)\right]=2 h D_{41} \sin ^{2}\left(k_{4} \delta_{4} / 2\right)$,

where $k_{j}=\frac{\omega}{v_{j}}$ is the wave vector.

$D_{41}$ coefficient is the complex constant which is included in the common solution of the wave equation for spreading the longitudinal bulk waves in the piezoelectric:

$D_{41}=\left[\left(F_{21} M_{1}-\tilde{\beta}_{1} \tilde{\beta}_{2} N_{1}\right)\left(\tilde{c}_{1} \tilde{s}_{2}+\xi \tilde{s}_{1} \tilde{c}_{2}\right)+\xi\left(n_{1}+m_{1} C_{11}\right)+\right.$

$+\left(F_{22} M_{2}-\widetilde{\beta}_{1} \tilde{\beta}_{2} N_{2}\right)\left(\tilde{c}_{1} \tilde{c}_{2}-\xi \tilde{s}_{1} \tilde{s}_{2}\right)-\xi \tilde{s}_{1}\left(F_{11} M_{1}-\tilde{\beta}_{1} N_{1}\right)-$

$-\tilde{c}_{1}\left(F_{12} M_{2}-\widetilde{\beta}_{1} N_{2}\right)+\left(-K_{1} C_{32}+\widetilde{\beta}_{1} \widetilde{\beta}_{2} \widetilde{\beta}_{3} L_{1}\right) \times$

$\times\left(\tilde{c}_{3}\left(\tilde{c}_{1} \tilde{s}_{2}+\xi \tilde{s}_{1} \tilde{c}_{2}\right)+\xi \tilde{s}_{3}\left(\tilde{c}_{1} \tilde{c}_{2}-\xi \tilde{s}_{1} \tilde{s}_{2}\right)\right)+\left(m_{1} C_{31}+n_{1} \tilde{\beta}_{1} \tilde{\beta}_{2} \tilde{\beta}_{3}\right) \times$

$\left.\times\left(\tilde{s}_{3}\left(\tilde{c}_{1} \tilde{s}_{2}+\xi \tilde{s}_{1} \tilde{c}_{2}\right)-\xi \tilde{c}_{3}\left(\tilde{c}_{1} \tilde{c}_{2}-\xi \tilde{s}_{1} \tilde{s}_{2}\right)\right)\right] /\left[-\tilde{c}_{4}\left(\tilde{c}_{3}\left(\tilde{c}_{1} \tilde{s}_{2}+\xi \tilde{s}_{1} \tilde{c}_{2}\right)+\right.\right.$ $\left.\left.+\xi \tilde{s}_{3}\left(\tilde{c}_{1} \tilde{c}_{2}-\xi \tilde{s}_{1} \tilde{s}_{2}\right)\right)\right]$

$C_{j 1}, C_{j 2}$ coefficients are complex constants which are included in the common solution of the equation system describing the spread of the temperature waves in the sample.

$$
\begin{aligned}
& C_{11}= {\left[R_{2} \tilde{\beta}_{1}\left(\gamma c_{2} c_{3}+s_{2} s_{3}\right)-R_{1} \tilde{\beta}_{1}\left(\gamma c_{3} s_{2}+c_{2} s_{3}\right)+\tilde{\beta}_{1} \tilde{\beta}_{2} \times\right.} \\
&\left.\times\left(-R_{2} \gamma c_{3}+R_{1} s_{3}\right)-C_{12}\left(\gamma c_{3}\left(s_{1} c_{2}+\gamma c_{1} s_{2}\right)+s_{3}\left(s_{1} s_{2}+\gamma c_{1} c_{2}\right)\right)+C_{31} \gamma\right] / \\
& \quad /\left[\gamma c_{3}\left(c_{1} c_{2}+\gamma s_{1} s_{2}\right)+s_{3}\left(c_{1} s_{2}+\gamma s_{1} c_{2}\right)\right]
\end{aligned}
$$

$$
C_{12}=\frac{\beta_{1} B_{1}}{\sigma_{1}}, \quad C_{31}=-B_{1} e^{\left(\beta_{1}-\beta_{2}\right) \delta_{1}} e^{-\beta_{1} \ell},
$$$$
C_{32}=\frac{\left.C_{31} \mid c_{3}\left(c_{1} s_{2}+\gamma s_{1} c_{2}\right)+s_{3} \gamma\left(c_{1} c_{2}+\gamma s_{1} s_{2}\right)\right]+C_{12} \gamma+R_{2} \tilde{\beta}_{1} \gamma s_{1}}{s_{3}\left(c_{1} s_{2}+\gamma s_{1} c_{2}\right)+\gamma c_{3}\left(c_{1} c_{2}+\gamma s_{1} s_{2}\right)}+
$$$$
+\frac{R_{1} \tilde{\beta}_{1} c_{1}-\tilde{\beta}_{1} \tilde{\beta}_{2}\left[R_{2}\left(c_{1} s_{2}+\gamma s_{1} c_{2}\right)+R_{1}\left(c_{1} c_{2}+\gamma s_{1} s_{2}\right)\right]}{s_{3}\left(c_{1} s_{2}+\gamma s_{1} c_{2}\right)+\gamma c_{3}\left(c_{1} c_{2}+\gamma s_{1} s_{2}\right)}
$$

$D_{41}$ and $C_{j 1}, C_{j 2}$ coefficients were determined substituting the common solution of the equations (1) - (7) in the boundary conditions (8) - (12).

All other coefficients included in formulae are the intermediate designations which were entered to avoid any unwieldy calculations:

$$
\begin{array}{ll}
\xi=\frac{c_{1}^{T} k_{1}}{c_{2}^{T} k_{2}} & \tilde{c}_{j}=\cos k_{j} \delta_{j} \\
\tilde{s}_{j}=\sin k_{j} \delta_{j} & \tilde{\beta}_{j}=e^{-\beta_{j} \delta_{j}} \\
c_{j}=\operatorname{ch} \sigma_{j} \delta_{j} & s_{j}=\operatorname{sh} \sigma_{j} \delta_{j}
\end{array}
$$




\section{E.V. Vertsanova, Yu.I. Yakimenko: Mathematical model of photoacoustic microscopy...}

$$
\begin{aligned}
\gamma= & \frac{\kappa_{1} \sigma_{1}}{\kappa_{2} \sigma_{2}} \quad R_{1}=B_{2}-\gamma B_{1} \\
R_{2} & =B_{2}-B_{1} \\
F_{11} & =C_{11} s h \sigma_{1} \delta_{1}+C_{12} c h \sigma_{1} \delta_{1} \\
F_{12} & =C_{11} c h \sigma_{1} \delta_{1}+C_{12} s h \sigma_{1} \delta_{1} \\
F_{21} & =-C_{31} s h \sigma_{1} \delta_{3}+C_{32} c h \sigma_{1} \delta_{3} \\
F_{22} & =C_{31} c h \sigma_{1} \delta_{3}-C_{32} s h \sigma_{1} \delta_{3} \\
M_{1} & =\frac{\kappa_{2} \sigma_{2} K_{1}-\kappa_{1} \sigma_{1} K_{2}}{\kappa_{2} \sigma_{2}} \\
N_{1} & =L_{1}-L_{2}+\frac{K_{2}}{\kappa_{2} \sigma_{2}}\left(\kappa_{2} \beta_{2} B_{2}-\kappa_{1} \beta_{1} B_{1}\right) \\
M_{2} & =\frac{c_{1}^{T}\left(\sigma_{1} K_{1}-\eta_{1} \alpha_{1}\right)-c_{2}^{T}\left(\sigma_{2} K_{2}-\eta_{2} \alpha_{2}\right)}{c_{2}^{T} k_{2}} \\
N_{2} & =\frac{c_{2}^{T}\left(\beta_{2} L_{2}-\eta_{2} \alpha_{2} B_{2}\right)-c_{1}^{T}\left(\beta_{1} L_{1}-\eta_{1} \alpha_{1} B_{1}\right)}{c_{2}^{T} k_{2}}- \\
& -\frac{c_{2}^{T}\left(\sigma_{2} K_{2}-\eta_{2} \alpha_{2}\right)\left(B_{2}-B_{1}\right)}{c_{2}^{T} k_{2}} \\
K_{j} & =\frac{\eta_{j} \alpha_{j} \sigma_{j}}{k_{j}^{2}+\sigma_{j}^{2}} \quad B_{j}=-\frac{\beta_{j} B_{j} \eta_{j} \alpha_{j}}{k_{j}^{2}+\beta_{j}^{2}} \\
\sigma_{j} & \left.=\sqrt{\frac{\omega}{2 \chi_{j}}(1+i)} \quad \beta_{1}^{2}-\frac{i \omega}{\chi_{j}}\right)
\end{aligned}
$$

\section{Conclusion}

The main result of this work is the reception of the expression for an open-circuit voltage on a piezoelectric transducer contacting acoustically with the optically nontransparent and thermally thick sample. It is obvious that despite the apparent simplicity, the resulting expression (20) is rather cumbersome and inconvenient to deter-

mine on its basis the amplitude and phase of the photoacoustic response that are really measured values. However, it is the advantage that this expression is the most full one among the solutions in literature for the case of optically non-transparent and thermally thick samples. As to its simplification in order to obtaine more compact expressions for both the spectral and frequency dependences of the amplitude and phase of the photoacoustic response as well as to develope the method for the determination of thermal characteristics, the problems were solved in details, for example, in $[1,5,6]$. The main task of this work was only to obtain the solution for the open-circuit voltage which would be perspective for the photoacoustic image processing.

\section{Summary}

Mathematical model of piezoelectrical detection of photoacoustic effect in opticaly semi-transparent and thermally thick solid objects with the undersurface inhomogeneity is proposed for the case of free binding of a piezo-sensor in a photoacoustic microscopy cell. The model gives an opportunity to estimate the influence of the undersurface defects on the photoacoustic response as well as to take into account the contribution of this factor into the output signal of the investigated objects.

\section{References.}

1. J. Jackson W., Amer N. Piezoelectric photoacoustic detection: Theory and experiment // J. Appl. Phys. — 1980. — 51, N 6. - P. 3343 - 3353.

2. Rosencwaig A., Opsal J. Thermal Wave Imaging with Thermoacoustic Detection // IEEE Transactions on ultrasonics, ferroelectrics and frequency control. - 1986. - 33, N 5. - P. 957 - 960 .

3. Mandelis A. Photoacoustic and thermal wave phenomena in semiconductor // Elsevier Science Publishing Co., Netherlands, Amsterdam, - 1987. — P. 471.

4. Green $R$. Nondestructive evaluation for materials characterization // Mat. Res. Soc. Symp. Proc. - 1989. - 142. - P. $23-29$.

5. Gulyaev Yu.V., Morozov A.I., Raevskii V.Yu. Photoacoustic spectroscopy of opticaly non-transparent objects with piezoelectrical detection (in Russian) // Acoustic journal. - 1985. - V. XXXI - issue 4. - P. $469-473$.

6. Blonskij I. V., Tkhoryk V. A., Shendeleva M. L. Thermal diffusivity of solids determination by photoacoustic piezoelectric technique // J. Appl. Phys. - 1996. - 79, N6. - P. $363-369$. 